\title{
Analysis Results from the Cosmic Ray Energetics And Mass Instrument for the International Space Station (ISS-CREAM)
}

\author{
Scott L. Nutter, ${ }^{a, *}$ Tyler Anderson, ${ }^{b}$ Yu Chen, ${ }^{b}$ Stephane Coutu, ${ }^{b}$ Tyler LaBree, ${ }^{a}$ Jason T. \\ Link, ${ }^{c, d, \dagger}$ John W. Mitchell, ${ }^{d}$ S. A. Isaac Mognet, ${ }^{b}$ Kenichi Sakai, ${ }^{c, d}$ Jacob Smith ${ }^{c, d}$ and \\ Monong $\mathbf{Y u}^{b}$ \\ ${ }^{a}$ Northern Kentucky University, Dept of Physics, Geology, and Engineering Technology, Highland Heights, KY \\ 41099 USA \\ ${ }^{b}$ Penn State University, Department of Physics, University Park, PA 16802, USA \\ ${ }^{c}$ Center for Research and Exploration in Space Science and Technology (CRESST), UMBC, Baltimore MD, 21250 \\ ${ }^{d}$ NASA Goddard Space Flight Center, Astroparticle Physics Laboratory, Greenbelt, MD 20771, USA \\ ${ }^{\dagger}$ No longer affiliated with NASA or CRESST as of May 2021. \\ E-mail: nutters@nku.edu
}

\begin{abstract}
We present the results of an analysis of on-orbit data from the Cosmic Ray Energetics And Mass instrument for the International Space Station (ISS-CREAM). The design objective is to measure the elemental spectra of cosmic rays from $Z=1$ to $Z=26$ over the energy range of $10^{12}-10^{15} \mathrm{eV}$. The instrument was installed on the ISS on August 22, 2017 with operations terminated on February 12, 2019 , resulting in approximately 1.5 years of operation. We compare detailed GEANT4 simulations to instrument data, demonstrate how we determine the appropriate absolute energy calibration for the instrument, and show some preliminary results.
\end{abstract}

$3^{\text {th }}$ International Cosmic Ray Conference (ICRC 2021)

July 12th-23rd, 2021

Online-Berlin, Germany

*Presenter 


\section{Introduction}

The ISS-CREAM instrument is a detector suite based on the CREAM instrument [1] designed for flight on the International Space Station (ISS). Its goal is to measure the composition and energy spectra of cosmic-ray nuclei from $10^{12} \mathrm{eV}$ to $>10^{15} \mathrm{eV}$ [2]. Recent measurements [3-5] of elemental spectra of cosmic rays from hydrogen to iron nuclei, at energies up to $\sim 10^{14} \mathrm{eV}$, have highlighted the need to refine our understanding of cosmic-ray production and acceleration, presumably in Galactic supernova remnants. The measured elemental cosmic-ray spectra follow a single power-law distribution in the range $10-100 \mathrm{GeV} / \mathrm{n}$, with a discrepant hardening of the spectra observed beginning around $200 \mathrm{GeV} / \mathrm{n}$. A detailed understanding of this effect is needed to improve modeling of the production and transport of galactic cosmic rays. ISS-CREAM data will add to the growing collection of spectral details for individual cosmic-ray nuclei.

We describe here the ongoing analysis efforts and present preliminary results. The analysis is characterized by strong agreement between on-orbit and simulated data, with multiple checks on absolute energy calibration and charge using on-orbit data. In particular, calibration of the ISS-CREAM calorimeter using on-orbit data indicates the energy deposition response should be increased by a factor of 30-40 over that of the balloon-borne CREAM calorimeter. Note that only the CREAM calorimeter and electronics were beam-tested before launch (see Section 2.1). While physically very similar to the CREAM version, The ISS-CREAM calorimeter is designed to have a greater dynamic range, and has different electronics from the calorimeter tested in the beam tests reported in [6].

\section{Instrument description and data processing}

The primary instruments on ISS-CREAM are the silicon charge detector (SCD) [7] to identify the charge, and the tungsten/scintillating fiber sampling calorimeter (CAL, 20 radiation lengths thick) to measure the energy of nuclei from $10^{12}-10^{15} \mathrm{eV}$. A densified carbon target $(0.43$ nuclear interaction length) is placed between the SCD and the CAL to initiate particle showers. In addition, the top and bottom scintillator-based counting detectors (TCD and $\mathrm{BCD}$ ), placed above and below the calorimeter, provide shower position $[8,9]$. At the bottom of the instrument stack, the boronated scintillator detector (BSD), provides an additional shower energy measurement in this analysis [10]. The TCD/BCD and BSD were included to discriminate electrons from protons through measurements of shower shape and neutron content, respectively. Readout is triggered by programmable algorithms using both the CAL and T/BCD. The full instrument is described in more detail in [11].

ISS-CREAM was launched in August 2017 on a SpaceX Falcon rocket in the trunk of the Dragon module and installed on the Japanese Experiment Module - Exposed Facility (JEM-EF) where it was operated until February 2019. For the first year, detector voltages were turned off during South Atlantic Anomaly (SAA) transit. After one year of operations, the instrument was always on, even through the SAA, except for a few short down-periods.

\subsection{Calibration}

Prototypes of the CAL, SCD, and BSD were exposed to test beams at CERN. The calorimeter design is nearly identical to that of the precursor balloon-borne experiment, CREAM, which was calibrated multiple times in electron and pion beams at CERN $[6,12]$. The calibrations were used to map ribbon to ribbon response differences and determine the conversion factor, measured in energy deposit/ADC 

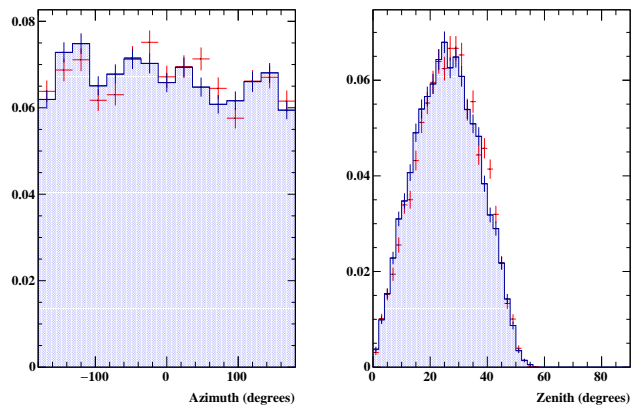

Figure 1: Azimuth (left) and zenith angle (right) distributions of simulated and on-orbit data, normalized to equal areas. Blue histogram: On-orbit data. Red points: Simulated data. Error bars are statistical.
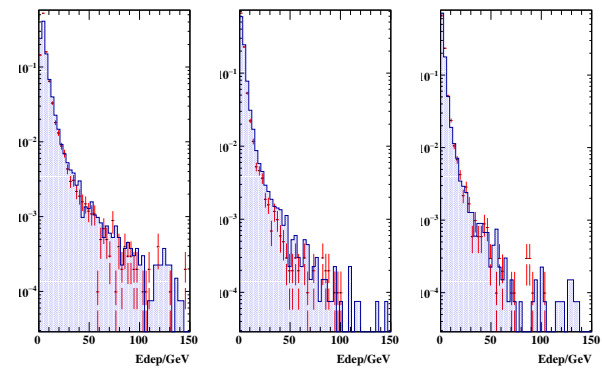

Figure 2: Calorimeter energy deposit for a nine ribbon sum (9RS) in events for selected simulated and on-orbit data for events above $\sim 1.7 \mathrm{TeV}$ (using energy calibration of [13]), normalized to equal areas. Left to right: Total, XZ, and YZ transverse view 9RS energy. Simulated data are in red, on-orbit data in blue. Error bars are statistical.

count. To determine this factor, the CAL response to electron showers at multiple energies from $75 \mathrm{GeV}$ - $250 \mathrm{GeV}$ is matched to GEANT4 simulations, yielding $0.09 \mathrm{MeV} / \mathrm{ADC}$. This conversion factor is then modified to account for the different electronics used on the ISS-CREAM calorimeter. Our validation of the ISS-CREAM CAL energy scale by comparing the CAL signal to the BSD signal, as described in [13], indicates the energy deposit per ADC count differs from that of the balloon instrument (CREAM) by a factor of $30-40$ (preliminary).

In all detectors except the BSD, a precision charge injection system measures individual electronics channel response, and is used to characterize and correct the slightly non-linear response and any time dependence, and ideally are used to connect the balloon and ISS calorimeter electronics conversion factors. In the SCD, particle charge peaks in on-orbit data are used to convert to charge Z. A channel-by-channel correction, based on the location of the carbon peak in each pixel, is also applied.

The ambiguity in the absolute energy scale arises from the change in the digitization electronics used for the ISS-CREAM CAL to extend dynamic range beyond that of the tested balloon-borne detector, resulting in a larger conversion factor. The ISS-CREAM conversion factor is based on some unvalidated assumptions regarding ADC response to the charge injection calibration system, and the conversion of DAC units to charge (pC). The conversion depends on several factors, including the charge per DAC, and the total charge input (DAC value) at which saturation of the ADC occurs. These factors must all be determined for both the balloon and ISS electronics. For example, a conversion factor based on the assumption of full scale values of electronics components results in a factor of 5 increase in the conversion factor from the beam calibration, to $0.45 \mathrm{MeV} / \mathrm{ADC}$. A recalculation using a number of measured DAC ranges and $\mathrm{ADC}$ responses can yield a conversion factor between 12 and 40 times the balloon value (1.1-3.6 MeV/ADC). We were uncomfortable with continuing to depend on inferring the absolute energy scale from the charge calibration systems, which have considerable leeway in interpretation, so took the time to develop this calibration based on the on-orbit data.

A definitive argument for the need for a careful study of the calorimeter calibration is based on the total number of particles detected for particular charge species. The effective instrument energy threshold 
is set by the trigger thresholds. Judging by the energy deposit at which the carbon flux reconstructed from a sum of 9 ribbons in each layer of the CAL centered on the track (9RS) assumes a power law shape, the instrument threshold can be identified. The observed number of carbon with energies greater than this threshold is $\sim 1000$. A calculation using the live time, geometric factor and efficiency of Section 4 suggests a trigger threshold $\sim 5.5 \mathrm{TeV}$, close to what is observed with the $\mathrm{x} 30$ energy calibration described in [13].

Data were sparsified in all detectors to reduce downlink bandwidth requirements, except the BSD, which has a fixed small number of readout channels. The sparsification level in the SCD is $\sim 1 / 8$ to $1 / 2$ of the proton peak, depending on channel. In the CAL, the sparsification level was $\sim 50 \mathrm{MeV}$, which is far above the mean energy deposit in a ribbon expected for a non-interacting iron nucleus, thus negating the possibility of using the usual on-orbit calibration with non-interacting ionizing particles (e.g. iron nuclei).

\section{Analysis Status}

Several tools were developed to assist in the analysis effort. These include a track-independent cosmic-ray identification using machine learning software [14], a detailed GEANT4-based instrument simulation, multiple tracking methods, and a unified ROOT-based event viewer, which simultaneously displays graphically all detector responses for both simulated and on-orbit events. The event viewer is extremely useful in identifying mapping issues and characterizing noise events. With the viewer, we identified and resolved issues with the original T/BCD channel to position maps, validated the machine-learning cosmic-ray identification tool, checked tracking algorithms, and explored noise event characteristics, for example. Exhaustive efforts to find a hidden inefficiency in the trigger system, or missing data from the CAL, did not find any evidence. The instrument functioned reasonably, as shown in [15]. Track-independent methods of identifying showering cosmic-rays (CRs) did not uncover additional events.

The data were down-selected for consistent and well-behaved periods of operation. Good data periods are defined to be those in which the CAL high voltages (HV) are set to their nominal values (plus the period in which one CAL HV supply is off to mitigate noise), the top SCD layer is fully functional, and the second layer is either fully or 3/4 functional. We choose not to analyze data during periods identified as particularly noisy, since the acquisition system was not tested under those conditions.

During SAA transit in the first year of operations, live time counters continued to accumulate while the acquisition system was left on but detector voltages were off. In addition, the functioning fractions of detectors changed sporadically, especially in the SCD. A configuration cut is thus used to identify those periods in which the instrument is in similar working configuration, and only live time accumulated during those periods is counted. The periods consist of combinations of two CAL configurations and two configurations of the top two SCD layers. The T/BCD and BSD were stable after the first $\sim$ month of operations.

\subsection{Simulations}

The instrument simulations are key in fully understanding and interpreting the data. The GEANT4 simulation consists of a detailed instrument model, down to the individual scintillating fibers in the CAL, and includes the correct small tilts of the SCD silicon. Care is taken to ensure that the simulated detector masses agree with measured values. A simulated input dataset from nuclear cosmic-ray species with power-law spectra and relative abundance normalization as described in [16] is treated identically to the data, going through the exact same analysis code. Various flight distributions of interest are directly comparable. For example, see Figures 1 and 2 for comparisons of reconstructed azimuth and zenith angle 
distributions and total CAL energy deposit between simulated and on-orbit data, respectively. For each set of histograms, simulated or on-orbit, data were normalized to equal area using the same normalization constant. The simulated and on-orbit data are analyzed identically, with the same code and cuts.

The nature of power-law spectra results in an ambiguity in checking the energy scale when comparing simulated to on-orbit data. Any multiplicative factor in the total energy scale applied to on-orbit data by increasing the CAL conversion factor can be duplicated in simulated data by the proper treatment of simulated trigger and sparsification thresholds. Energy conversion factors from 2.5 to 72 times the balloon value have been matched to data, illustrating the challenge in setting the absolute CAL energy response scale.

\subsection{Particle Tracking and Charge Identification}

A particle track is necessary to point back to the SCD to determine particle charge in order to mitigate charge misidentification due to backsplash from interactions in the carbon targets. We explored a number of different methods of finding a particle track, and found that a tracking method that combines information from the CAL, TCD and SCD is most effective. The method (see [15]) iteratively constructs a track, starting with the energy-deposition-weighted positions in the two CAL views, the TCD photodiode position with the maximum signal, and the highest SCD signal. Simulated and on-orbit tracking distributions of azimuth and zenith angle distributions are in very good agreement, as shown in Figure 1.

The track is extrapolated to the location of the top two SCD layers. A circle of confusion of radius $70 \mathrm{~mm}$ centered on the track intersection with each layer is used to limit the region of interest for charge searching, and the highest signal within the circle is used to determine particle charge. The SCD provides two particle charge measurements, one in each of the top two layers. The charge distribution is shown in Figure 3. Note the clear charge peaks for elements from hydrogen to iron, and the boron, sulfur, argon, and calcium peaks. Table 1 contains the peak and standard deviation of Gaussian fits to visible charges (Landau convolved with Gaussian for $\mathrm{H}$ and $\mathrm{He}$ ). The fit parameters are used in a $2 \sigma$ charge selection. The analysis selects events from this distribution for a reconstructible high energy.

\subsection{Energy Determination}

To mitigate noisy channel contributions to the measured total energy deposit in the CAL, the track is used to define a nine ribbon wide pathway (nine ribbon sum, 9RS), with the center ribbon position determined by the fit track. The energies of all ribbons whose centers are within that path are added together to get the energy deposit in each tracking view as well as the total energy deposit. Studies show that the CAL response is equivalent when choosing widths above a five ribbon sum, up to the maximum tested 9RS, but total energy deposition in the on-orbit data results in a significantly different distribution from the simulated data, which we interpret as the inclusion of noise channel data in the sum. The energy deposit of each view is corrected for track length along the fiber length in the transverse view since the fibers present a round cross section in the tracking view. The corrected energy deposit from each view is then summed to form the total 9RS energy deposit for each event. Figure 2 shows a comparison of the simulated and on-orbit data angle-corrected 9RS energy deposit.

Primary particle energy reconstruction is based on the simulated CAL and BSD energy deposits compared to the primary total kinetic energy. For the CAL, a second order polynomial is fitted to the simulated data response, then inverted. Note that simulated data are treated identically to on-orbit data in finding the energy reconstruction relationship, with the same cuts and tracking. This method allows reconstruction 
of the power-law spectral index within 3\% above $1 \mathrm{TeV}$ total energy for simulated carbon power-law data. The slope of energy deposit in the calorimeter versus incident energy is found to be $1.70 \mathrm{GeV} / \mathrm{TeV}$. Using the 12 BSD late light signals after response map and angle corrections, the energy reconstruction is linear with a slope of $0.131 \mathrm{TeV} / \mathrm{MIP}$. Studies are still needed to map out the relationship at the highest energies.

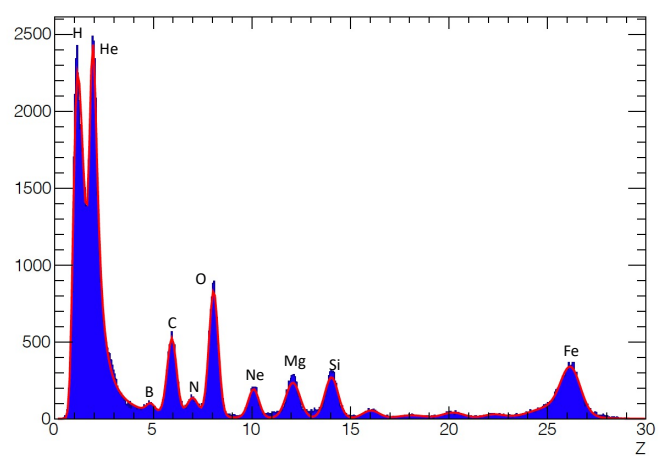

Figure 3: Charge distribution from the SCD using the charge identification method described in the text. The relative abundances (peak heights) are not physically interpretable in this figure without additional corrections.

\begin{tabular}{l|c|r} 
Charge (Z) & Peak (e) & Width (e) \\
\hline Hydrogen (1) & 1.15 & 0.39 \\
Helium (2) & 1.90 & 0.28 \\
Boron (5) & 4.89 & 0.12 \\
Carbon (6) & 5.95 & 0.32 \\
Nitrogen (7) & 6.93 & 0.20 \\
Oxygen (8) & 8.05 & 0.24 \\
Neon (10) & 10.10 & 0.27 \\
Magnesium (12) & 12.10 & 0.36 \\
Silicon (14) & 14.00 & 0.34 \\
Sulfur (16) & 16.00 & 0.41 \\
Calcium (20) & 20.20 & 0.61 \\
Iron (26) & 26.20 & 0.51
\end{tabular}

Table 1: Peaks and widths from a simultaneous fit to the charge distribution of Figure 3.

\section{Fluxes}

The fluxes (for comparison only) of protons, helium, carbon, oxygen, and iron are calculated using the energy deposits in both the CAL and the BSD to determine primary particle kinetic energy and compared in Figure 4, for the $\mathrm{x} 30 \mathrm{CAL}$ conversion factor results. The calculation is

$$
\Phi(G e V)=\frac{N}{\epsilon} \times \frac{1}{S \Omega T}
$$

where

$\Phi$ is the flux in $\left(\mathrm{m}^{2} \mathrm{sr} \mathrm{s} \mathrm{GeV}\right)^{-1}$,

$N=f\left(d E / d x \rightarrow E_{\text {kin }}\right)$ is the number of particles detected per $\mathrm{GeV}$ using the function mapping energy deposition to total particle kinetic energy for the CAL or BSD,

$\epsilon$ is the net efficiency, $42 \%$, determined with simulated data for track and charge identification, and charge selection,

$S \Omega$ is the geometric factor, $\sim 0.35 \mathrm{~m}^{2} \mathrm{sr}$, which varies by particle, trigger, and energy, and $T$ is the live time, 162.78 days.

The total live time estimate must account for periods during which the instrument was not in a configuration for particle detection. It can be estimated in two ways. First, we use the live time counter and only add up good configuration periods, where this counter is reported in the HK data stream every $\sim 12$ seconds, separate from the data, and time stamps are used to correlate events with live time. The 
second method is to add up time elapsed between consecutive events that occur during good configuration periods and are within 300 seconds of each other. These two methods differ by $15 \%$, and the live time is a major source of systematic error at this time.

Event selection efficiencies are determined with simulated data. Proton and helium selection is more sensitive to backsplash and noise channels, and selection cuts are still being studied. Efficiencies due to tracking, correct charge identification, and the $2 \sigma$ charge selection result in a $42 \%$ overall efficiency. The on-orbit data is likely not as efficient, and the exact experimental efficiencies are still being determined. In addition, obscuration due to the JEM and other ISS structures is uncertain.

\section{Conclusions}

On-orbit calibration between the CAL and BSD compared to simulated data strongly supports a 30-40 increase in the CAL energy deposit over the CREAM version of the calorimeter electronics. The fluxes are in good agreement with those reconstructed from the BSD, and show fair agreement with the reference fluxes, depending on particle species.

The displayed fluxes can be moved vertically on the plots by finding further inefficiencies, and horizontally with energy scaling. This analysis applied to simulated data results in equivalent CAL and BSD fluxes, indicating the problem is not in the analysis method. The absolute energy calibration factor of 30-40 suggested by the BSD study brings the CAL and BSD fluxes into alignment for the onorbit data. Future work will center on refining the BSD calibration of the CAL, properly combining the different detector configurations in simulated data, checking the live time and efficiency calculations, and studying systematic errors. For the efficiencies, the multiple tracking algorithms allow
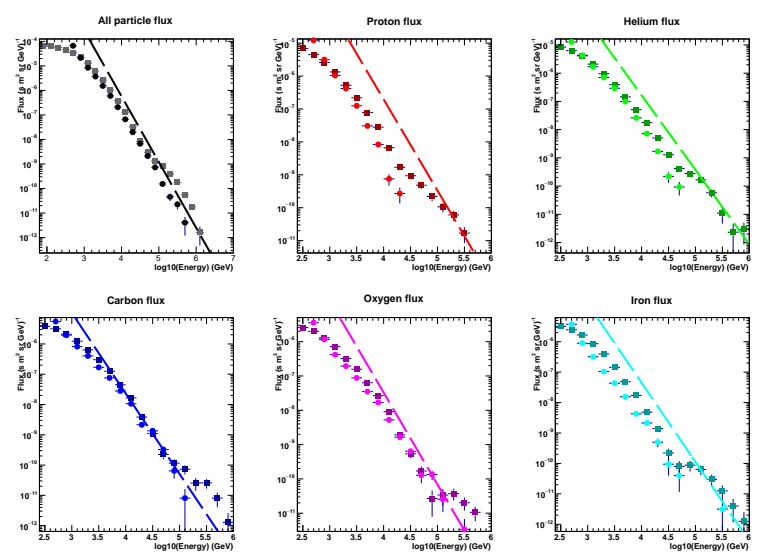

Figure 4: Flux in $\left(\mathrm{m}^{2} \text { sr s GeV) }\right)^{-1}$ vs total particle kinetic energy calculated with the CAL (circles) and BSD (squares) energy deposit as described in the text using a conservative $\mathrm{x} 30$ scaling of the CAL energy deposits. Open circles use the original CAL scaling as described in the text. Errors are statistical. Left to right, top row: all particles, protons, and helium. Bottom row: carbon, oxygen, and iron. The dashed lines are reference spectra from [16]. Results are for illustration of the conversion factor only. us to estimate individual detector efficiencies by using tracking that does not depend on the detector in question.

\section{Acknowledgments}

This work was supported in the U.S. by NASA grants NNX17AB43G, NNX17AB42G, and their predecessor grants, as well as by directed RTOP funds to NASA GSFC. The authors also thank M. Geske, Penn State, for contributions to the BSD, and K. Wallace at Northern Kentucky University for contributions to Monte Carlo simulations. We also recognize the contributions of past CREAM and ISS-CREAM collaborators. 


\section{References}

[1] H. S. Ahn et al., The Cosmic Ray Energetics And Mass (CREAM) instrument. Nucl. Instrum. Methods A, 579, 1034-1053, 2007.

[2] Y. Amare, et al., ISS-CREAM Collaboration, The Cosmic Ray Energetics And Mass Instrument on the International Space Station (ISS-CREAM), in preparation, to be submitted to Nucl. Inst. and Meth. A (2021)

[3] H.S. Ahn, et al., CREAM Collaboration, Discrepant hardening observed in cosmic-ray elemental spectra, Astrophys. J. 714 (2010) L84

[4] O. Adriani, et al., PAMELA Collaboration, PAMELA measurements of cosmic-ray proton and helium spectra, Science 332 (2011) 69

[5] M. Aguilar, et al., AMS Collaboration, Properties of elementary particle fluxes in primary cosmic rays measured with the Alpha Magnetic Spectrometer on the ISS, Proc. of the 35th Int. Cosmic Ray Conf., Busan, South Korea (2017)

[6] H. G. Zhang, D. Angelaszek, M. Copley, J. H. Han, H. G. Huh, Y. S. Hwang et al., Performance of the ISS-CREAM calorimeter in a calibration beam test. Astroparticle Physics 130 (2021) 102583.

[7] J. Lee, et al., The ISS-CREAM Silicon Charge Detector for identification of the charge of cosmic rays up to $Z=26$ : Design, fabrication and ground-test performance, Astroparticle Physics 112 (2019) $8-15$.

[8] H.J. Hyun, et al., Performances of photodiode detectors for top and bottom counting detectors of ISS-CREAM experiment, Nucl. Inst. and Meth. A 787 (2015) 134.

[9] S.C. Kang, et al., On-orbit Performance of the Top and Bottom Counting Detectors for the ISS-CREAM Experiment on the International Space Station, Adv. in Space Research 64 (2019) 2564-2569.

[10] Y. Amare et al., The boronated scintillator detector of the ISS-CREAM experiment, Nucl. Inst. and Methods A 943 (2019) 162413.

[11] E.S. Seo, et al., Cosmic Ray Energetics And Mass for the International Space Station (ISS-CREAM), Advances in Space Research 53 (2014) 1451 - 1455.

[12] H.G. Zhang, et al., Performance of the ISS-CREAM calorimeter in a calibration beam test, Astroparticle Physics 130 (2021) 102583.

[13] Yu Chen, et al. On-Orbit Energy Calibration of the Calorimeter on the ISS-CREAM Instrument Using the Boronated Scintillator Detector. Poster 866, 37th International Cosmic Ray Conference (ICRC2021).

[14] Monong Yu, et al. Machine learning applications on event reconstruction and identification for ISS-CREAM. Poster 476, 37th International Cosmic Ray Conference (ICRC2021).

[15] Kenichi Sakai, et al. ISS-CREAM detector performance and tracking algorithms. Poster 1051, 37th International Cosmic Ray Conference (ICRC2021).

[16] B. Wiebel-Sooth, P. L. Biermann, and H. Meyer, Cosmic rays. VII. Individual element spectra: prediction and data, Astronomy and Astrophysics, v.330, p.389-398 (1998). 